\title{
Fluid-Dynamic Characterization and Efficiency Analysis in Plastic Separation of the Hydraulic Separator Multidune
}

\author{
Floriana La Marca, Monica Moroni and Antonio Cenedese \\ DICEA - Sapienza University of Rome, Rome
}

Italy

\section{Introduction}

Recovery of useable plastics from post-consumer and manufacturing waste remains a major recycling challenge. The global consumption of plastics was reported to be 230 million tonnes in 2005 (PlasticsEurope 2007a) of which 47.5 million tonnes were produced in Europe (25 European Union countries + Norway and Switzerland). Of the European production, only 22 million tonnes were reported as having been collected. Of this collected waste, 4 million tonnes were recycled as a manufacturing feedstock $(18 \%)$ and 6.4 million tonnes went into energy recovery $(29 \%)$, with the balance (11.6 million tonnes) probably being disposed in landfills (PlasticsEurope 2007b).

The recycling of plastics is a process essential to reduce the efflux of materials to landfills and to decrease the production of raw materials. In recent years awareness of the importance of environmental protection has led to the development of different techniques for plastic recycling. One issue related to the recycling of this material is the presence in the market of many types of plastics (polymers with additives), often with similar characteristics that make them difficult to differentiate in the recovery phase.

The separator "Multidune" is a hydraulic separator by density. Its name derives from the characteristic undulate profile of the channel where separation occurs. The channel is constructed from a sequence of closed parallel cylindrical tubes welded together in plane which are then sliced down the lateral mid-plane and the lower complex is laterally shifted relative to the upper complex. The Multidune allows solid particle separation according to their specific weight and the velocity field establishing within the apparatus.

Previous investigations (De Sena et al., 2008; Moroni et al. 2008) suggested the flow within the Multidune is organized into three main patterns. Principally, a longitudinal transport flow takes place, where the velocity is high. A particle belonging to this region can move from one camera to another. The second region is the lower recirculation zone with high values of the vorticity field. Particles belonging to this region undergo the vertical impulse of the fluid. The thrust is proportional to the vertical velocity component and, in conjunction with gravity and buoyancy, determines the destiny of a particle. If the thrust is larger than the net weight of the particle, an interaction with the principal transport flow occurs and, consequently, the particle will move to the following chamber. The third region is the upper recirculation zone whose dimensions are smaller than the other recirculation zone. If a 
particle moves from the principal flow to the secondary vorticity zone, it will have the chance to come back to the previous chamber, assuming the principal transport flow thrust does not prevent it from falling out.

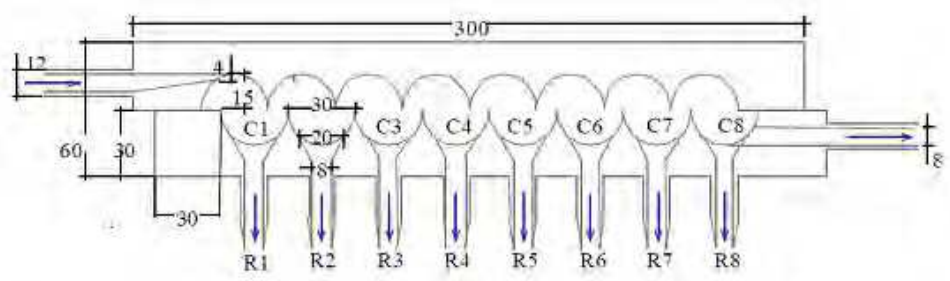

(a)

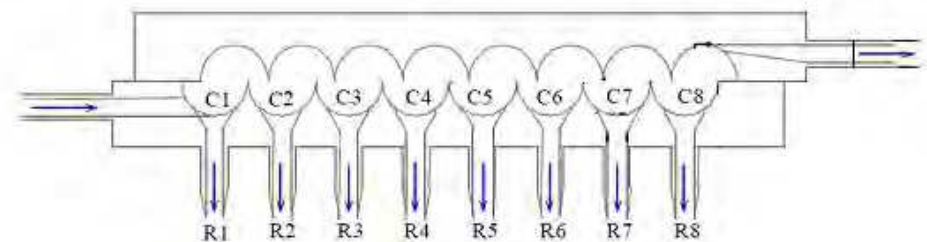

(b)

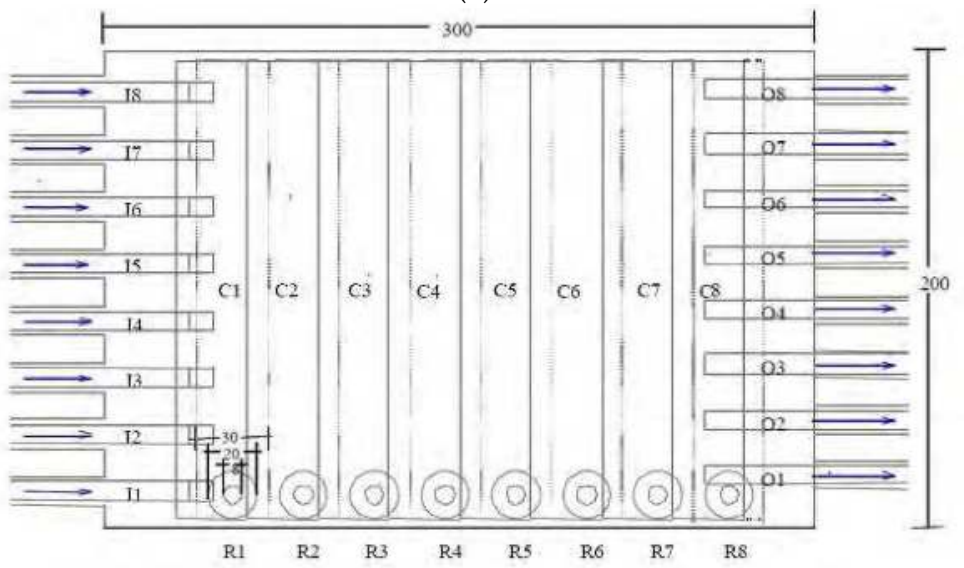

(c)

Fig. 1. Longitudinal section (dimensions in $\mathrm{mm}$ ) for Arrangement (a) A and (b) B; (c) top view of the apparatus. $C$ indicates the 8 chambers, $R$ the 8 collection nozzles, I the 8 inlet nozzles and $\mathrm{O}$ the 8 outlet nozzles. Dimensions for Arrangement $\mathrm{B}$ are the same as for Arrangement A

Because of the role played by velocity, the fluid dynamic investigation of the Multidune apparatus is a preliminary step to carry out in order to investigate its capability in separating solid particles. For this reason a novel experimental campaign was set up and image analysis was employed to detect the velocity field within the apparatus. Two different arrangements of the Multidune were employed, hereinafter Arrangement A and 
Arrangement B. Figure 1 presents a sketch of the longitudinal sections for the two arrangements, the top view and the apparatus dimensions in $\mathrm{mm}$.

Two sets of experiments were run. The camera imaged all chambers of Arrangement A and $B$ respectively and a passive tracer, i.e., a neutrally buoyant seeding particulate, was used to seed the fluid and reconstruct the fluid velocity field.

The fluid-dynamic investigation deals with the detection of several quantities of interest:

- mean velocity field and velocity profiles along the apparatus

- two-dimensional turbulent kinetic energy

- $\quad$ vorticity field

Generally, the detection of vortical structure (or coherent structure) immersed within a turbulent flow field is not straightforward. This book chapter will deal with this issue as well. Starting from the velocity gradient tensor, a topological analysis will be carried out with the aim of determining the critical points within the domain and to better represent areas with elevated values of the vorticity field.

The fluid-dynamic characterization will be correlated with the results of separation tests, carried out at lab-scale to separate particles of different plastic materials, characterized by low values in specific weight (about $1 \mathrm{~g} / \mathrm{cm}^{3}$, close to water specific weight).

\section{Topological description of a fluid-dynamic field}

It is usually difficult to individuate vortices, or coherent structures, within a statistical field which represents the background turbulent phenomenon. The detection of those structures is strictly related to their definition and it is influenced by the fact that the flow field can not be monitored at all scales. If an experimental monitoring technique is employed, a further limit is related to the availability of point, mono-dimensional, or twodimensional measures.

Among the principal methods employed to detect vortices, visualization is the first and the currently most employed. The fluid motion can be visualized through the injection of dye or tracer particles. Remarkably, the surface where vorticity assumes a constant value does not necessarily match the surfaces where the dye concentration is constant. Even if the Schmidt number $\left(\mathrm{S}_{\mathrm{c}}=\mathrm{v} / \mathrm{D}\right.$, where $\mathrm{v}$ is the kinematic viscosity and $\mathrm{D}$ the dye molecular diffusivity) is unitary, unlike the scalar behavior, vortices are subject to vortex stretching. Tracers would then present lower concentrations in areas of larger vorticity, and will not highlight fundamental behaviors of vortical dynamics. This inconvenient can be avoided seeding the fluid locally and detecting its behavior at distance and/or time intervals small respect to the characteristic scales (Joeng and Hussain, 1995).

Although the concept of vorticity is one of the most widely used in fluid mechanics, even today there is no generally accepted definition of vortex. A vortex is usually defined as the region of space enclosed by a surface formed by swirling lines (tangent at every point of the velocity curl). This definition is ambiguous as it would lead to identify a vortex within a laminar channel or in a Poiseille flow.

The conditions necessary for a proper definition of the vortex are (Hussain, 1986):

- $\quad$ inside the vortex, the vorticity $(\nabla \times \mathbf{u})$ must assume non-zero values, it is a necessary but not sufficient condition;

- $\quad$ the existence of the vortex must be identified by a scalar quantity;

- identification criteria must be invariant under Galilean transformations. 
Since the concept of vortex is associated with rotation of the fluid, some authors have proposed to identify a vortex where the pressure reaches the minimum required to balance the centripetal force. This definition falls in some situations, for example, in a convergent a minimum of pressure exists unless the fluid is characterized by a rotating motion.

Similarly, the use of trajectories and streamlines to identify a vortex does not appear appropriate since both are not invariant with respect to a Galilean transformation.

Some criteria for identifying vortical structures that meet the requirements above use scalar quantities derived from the velocity gradient tensor, $\nabla \mathbf{u}$ or $\mathrm{A}_{\mathrm{ij}}$ which is decomposed in its symmetric $S_{i j}$ and antisymmetric $W_{i j}$ parts. In particular, the eigenvalues of the velocity gradients are examined. In particular, the three criteria are based on:

- identification of regions where eigenvalues of $\nabla \mathbf{u}$ are complex numbers

- identification of regions where the second eigenvalue of tensor $S_{i k} S+W_{i k} W_{i k}$ is negative (eigenvalues sorted in descending order)

- identification of the regions where the second invariant of the velocity gradient tensor is positive.

Those three criteria are equivalent in a two-dimensional case, as the case under investigation.

\section{Experimental set-up}

The Multidune apparatus, $0.30 \times 0.20 \times 0.06 \mathrm{~m}^{3}$ in size, is composed by a sequence of closed parallel cylindrical tubes welded together in plane. The device is sliced down its lateral midplane and the lower half is shifted laterally and then fixed relative to the upper half. Arrangements A and B (Figure 1) are shaped by two different positions of the lower half. In Arrangement A, the lower half is shifted $0.012 \mathrm{~m}$ right, while it is shifted $0.012 \mathrm{~m}$ left for Arrangement $\mathrm{B}$. Each resulting chamber is labelled according to its position along the flow direction [C1 (first chamber), C2, .., C8 (last chamber)]. The first chamber has eight round input nozzles (I1, I2, ... I8) held at constant head ranging between $0.84 \mathrm{~m}$ and $2.69 \mathrm{~m}$. The last chamber has 8 round output nozzles $(\mathrm{O} 1, \mathrm{O} 2, \ldots, \mathrm{O} 8)$. Flow is induced in the lateral direction normal to the axis of the tubes by cutting inlet nozzles on one side of the device and outlet ones on the opposite side. Settled materials may be collected in the lower part of each half-cylinder.

The Multidune feeding occurs through a tank whose output pipe is split into 8 to distribute water within the apparatus through the 8 inlet nozzles. An overflow exit allows controlling the water level in the tank. The average flowrate within the Multidune apparatus will rely both on hydraulic head at the inlet nozzles and on the number of open outlet nozzles. With both arrangements, the experiments were run for three elevations of the tank (referred to the inlet nozzles height): $0.84 \mathrm{~m}, 1.84 \mathrm{~m}, 2.69 \mathrm{~m}$. Three outlet nozzles (O2, O4 and O6) were open when Arrangement A was adopted. Table 1 reports the difference in fluid elevation between the tank and the middle of inlet nozzles and the corresponding flowrates. Preliminary tests conducted with Arrangement B suggested the flowrate determined by opening three outlet nozzles was too large to allow plastic particles separation. Then experiments have been run with only one opened outlet nozzle (O3). In this case, the flowrates establishing within the apparatus are consistently lower.

Experimental results of separation of both mono- and multi-material samples (Moroni et al. 2011; La Marca et al. 2011) suggest the behaviour of particles introduced within the Multidune apparatus strongly depends on the characteristic velocity within the apparatus, 
dimension and density of plastic particles. The geometry of the channel and the type of working fluid are considered to play a key role in the process of separation. We then introduce the non-dimensional parameters:

\begin{tabular}{|c|c|c|c|c|c|}
\hline & $\begin{array}{c}\text { Difference in } \\
\text { fluid elevation } \\
\text { between tank } \\
\text { and Multidune }\end{array}$ & $\begin{array}{c}\text { Average } \\
\text { flowrate for } \\
\text { Arrangement } \\
\text { A }\end{array}$ & $\begin{array}{c}\text { Re } \\
\text { for } \\
\text { Arrangement A }\end{array}$ & $\begin{array}{c}\text { Average } \\
\text { flowrate for } \\
\text { Arrangemen } \\
\text { t B }\end{array}$ & $\begin{array}{c}\text { Re } \\
\text { for Arrangement } \\
\text { B }\end{array}$ \\
\hline & $(\mathrm{m})$ & $(1 / \mathrm{min})$ & - & $(1 / \mathrm{min})$ & - \\
\hline Q1 & 0.84 & 9.35 & 584.38 & 4.57 & 380.58 \\
\hline Q3 & 1.84 & 11.82 & 738.75 & 5.00 & 416.83 \\
\hline Q5 & 2.69 & 13.53 & 845.63 & 6.55 & 545.58 \\
\hline
\end{tabular}

Table 1. Difference in fluid elevation between the tank and the middle of the Multidune inlet nozzles and corresponding flowrates for both Arrangements.

- $\quad$ particle Froude number

$$
\theta=\frac{\mathrm{V}^{2}}{\frac{\rho_{\mathrm{s}}-\rho}{\rho} \mathrm{gd}}=\frac{\mathrm{V}^{2}}{\mathrm{~g}^{\prime} \mathrm{d}}
$$

where:

$\mathrm{V}$ : working fluid (water in the present case) characteristic velocity

$\rho_{\mathrm{s}}$ : particle density

$\rho$ : water density

g: acceleration of gravity

d: particle diameter

$\mathrm{g}^{\prime}$ : buoyancy parameter

- Reynolds number

$$
\operatorname{Re}=\frac{\mathrm{VH}}{\mathrm{v}}
$$

where:

$\mathrm{H}$ : apparatus characteristic dimension, set equal to the cylinder radius, i.e. $1.5 \mathrm{~cm}$

v: working fluid kinematic viscosity

Re for the three tank heights and both arrangements are reported in Table 1.

The following procedure was adopted to carry out experimental tests aimed at reconstructing the velocity field and separating the plastic materials:

1. set up of water tank height and hydraulic head;

2. water feeding in the apparatus through the 8 input nozzles;

3. saturation of the apparatus with water;

4. opening of the chosen output nozzles (O2, O4 and O6, in Arrangement A; O3 in Arrangement B);

5. passive tracer or sample feeding in the Multidune apparatus through the I3 e I4 input nozzles;

6. test execution, about three minutes; 
7. only for separation tests, recovery of material expelled from the output nozzle(s);

8. closing of the output nozzle(s);

9. only for separation tests, recovery of material settled in each chamber;

10. weighting of recovered materials after 24 -h drying.

\section{Fluid-dynamic characterization by image analysis}

The aim of the fluid-dynamic characterization of the Multidune apparatus was to reconstruct trajectories of tracer particles seeding the fluid under investigation and the velocity field evolution with time by means of image analysis techniques.

The need for measuring velocity fields has historically led researchers to develop experimental techniques and related instrumentation. An 'ideal' measurement system should be non intrusive to avoid flow field perturbations, should not require calibration, and should be suitable for obtaining the velocity field with a time and space resolution smaller than the characteristic time and length scales (i.e. Kolmogorov scale for turbulent flows). Velocity measurements based on optical methods capable of providing the velocity of tracer particles illuminated by a light source represent the best approximation of this 'ideal' system. The requirement is the working fluid to be seeded with neutrally buoyant particles which are assumed to follow the flow. The velocity vector is evaluated from the ratio of the tracer displacement, $\Delta s$, and the time interval $\Delta t$ required for the displacement to take place. The time interval must be small enough for the approximation to be reasonable.

Digital images were acquired using a high-speed high-resolution ( $1280 \times 1024$ pixels) camera at a rate of 250 frames per second and stored for analysis. The camera axis was set perpendicular to the Multidune lateral face. A high powder lamp produces a light sheet for illuminating the interior of the channel. Green plastic power (mean diameter of about 200 $\mu \mathrm{m})$ preconditioned with a solution of water and sodium hydroxide was used as the tracer for particle tracking. Preconditioning was used to neutralize the electrostatic charge on the particles. Tracer injection within the apparatus and data collection were started after the fluid reached steady state.

Feature Tracking (FT) was employed as the image analysis technique, i.e. a Particle Tracking algorithm which allows ignoring the constraint of low seeding density, being able to provide accurate displacement vectors even when the number of tracer particles within each image is very large (Moroni and Cenedese, 2005). FT reconstructs the displacement field by selecting image features (image portions suitable to be tracked because they remain almost unchanged for small time intervals) and tracking these from frame to frame. The matching measure used to follow a feature (and the $\mathrm{L} \times \mathrm{H}$ window around the feature, where $\mathrm{L}$ and $\mathrm{H}$ are the horizontal and vertical dimensions respectively) and its "most similar" region at the successive times is the "Sum of Squared Differences" (SSD) among intensity values: the displacement is defined as the one that minimizes the SSD. In Feature Tracking one applies the algorithm only to points where the solution for the displacement exists: those points are called "good features to track" (Shi and Tomasi, 1994). FT allows a Lagrangian description of the velocity field providing sparse velocity vectors with application points coincident with large luminosity intensity gradients (likely located along tracer particles boundaries). Lagrangian data are then used to reconstruct instantaneous and time-averaged Eulerian velocity fields through a resampling procedure. 
Fig. 2a and Fig. $2 \mathrm{~b}$ show the trajectories reconstructed by the FT algorithm within chambers C3 and C4 for experiments run with Arrangements A and B and difference in fluid elevation between the tank and the center of the Multidune inlet nozzles set to Q3.

Arrangement A

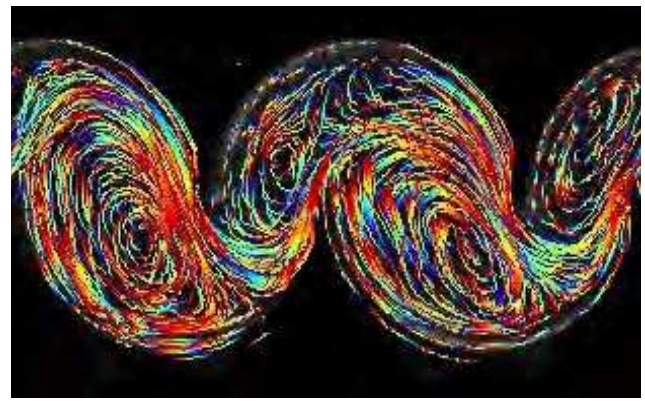

\section{Arrangement B}

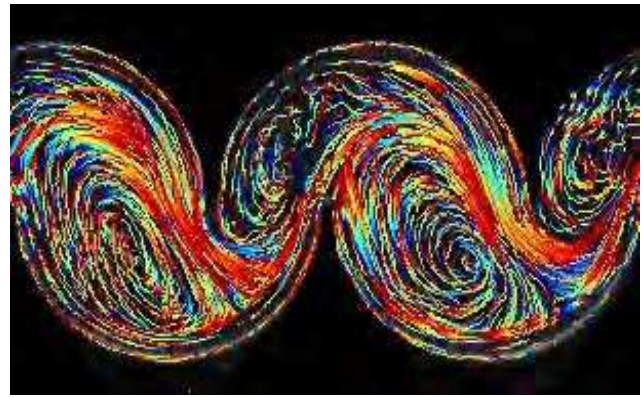

Fig. 2. Trajectories reconstructed by the Feature Tracking algorithm within chambers $C 3$ and C4 for experiments run with Arrangement A (a) and Arrangement B (b) and difference in fluid elevation between the tank and the center of the Multidune inlet nozzles set to Q3

The trajectories are visualized overlapping 32 consecutive positions of the tracer particles. The colours range from blue (associated with the first time shown) to red (associated to the last one). The comparison between the two images evidences the increased length of the trajectories reconstructed when three nozzles are open instead of one. At the higher flowrate, the movement of tracer particles from one frame to the next is expected to be larger. The trajectories qualitatively describe the velocity field within the apparatus and confirm the presence of three sectors, i.e. the principal current which appears more consistent with Arrangement B, the lower recirculation area, larger with Arrangement A, and the upper recirculation area bigger with Arrangement B.

Figure 3 and Figure 4 present the velocity vectors overlapped to the colormap of, respectively, the horizontal and vertical velocity components within chambers C3 and C4, both arrangements and differences in fluid elevation between the tank and the center of the Multidune inlet nozzles set to Q1, Q3 and Q5.

The analysis of both velocity fields and streamlines (Figure 5) suggests the fluid-dynamic behaviour of the Multidune apparatus for both arrangements is characterized by three predominant areas.

The principal transport flow. It is characterized by a positive value of the velocity component along the $x$ axis in the entire longitudinal section. The principal current is responsible for the transport of particles from one chamber to the next one and eventually it drives material to the outlet nozzles without separation. In Figure 5, it is indicated with red streamlines.

The lower recirculation zone. It is visible below the principal current, in each of the height chambers. The clock-wise rotating motion is suitable for subtracting particles from the principal current. It is expected that captured plastic particles will behave in one of the following ways: 
- $\quad$ settle within the chamber if sufficiently heavy;

- follow the upward portion of the rotating motion without reaching again the principal current being too heavy to perform a complete rotation;

- $\quad$ execute a complete rotation and being captured again by the principal transport flow to settle in the next chamber or be expelled.

The particle behavior within the recirculation zone is influenced by its density and dimension as well as by the presence of a vortex. In Figure 5, it is indicated with green streamlines.

The upper recirculation zone. It develops behind the principal transport current. The purpose of the upper recirculation zone is to subtract particles from the principal current and to transfer them backward. To do so they have to pass across the principal current and to settle in the chamber. The particle physical attributes and the characteristic velocity of the principal current will influence the efficacy of this process. In Figure 5, it is indicated with blue streamlines.

The fluid vein characterizing the principal current is constituted by an inner part with velocity increasing with the hydraulic head at the apparatus inlet. Nevertheless, the characteristic dimension of the principal current remains practically constant even varying the flowrate. It appears more consistent with Arrangement B. At each flowrate, the upper recirculation zone developing in the Multidune- Arrangement A presents values of the both components of velocity field significantly lower than the characteristic velocity of the principal current. For this reason, this zone results ineffective in capturing particles from the principal current and then in the separation process. It was expected that reducing the flowrate and/or modifying the inner geometry of the apparatus, the upper recirculation zone would have played a role in the separation process. This is confirmed by the experiments with the Multidune- Arrangement B. Reducing the flowrate, the velocity of the principal current decreases whereas it remains practically constant within the recirculation areas becoming comparable. Furthermore, the modified geometry favours the formation of a larger upper recirculation area in each chamber incrementing the contact surface with the main current and the probability of material exchange between the two zones.

The velocity field in the lower recirculation area is in each case significantly lower than the characteristic velocity in the principal current. With the Multidune- Arrangement A, the velocity within the lower recirculation zone increases rising the flowrate, especially in the ascending portion of the vortex. This is actually counterproductive in terms of separation, because of the drag of settled particles toward the principal current. A similar behaviour with analogous consequences characterizes the descending portion of the upper recirculation area establishing in the Multidune- Arrangement B.

Noticeably, the analysis of the fluid-dynamic field developing within the Multidune apparatus with both arrangements suggests the increase of the hydraulic head augments the transport effectiveness of the main current without improving the capture feasibility of both the upper and lower recirculation zones. The apparatus will then lose its effectiveness in separating plastic particles increasing both the hydraulic head and the transiting flowrate. Furthermore, with both arrangements, C3 and C4 present analogous recirculation areas. The same circumstance occurs at each flowrate establishing in the apparatus. Furthermore, an analogous behavior characterizes $\mathrm{C} 5$ and $\mathrm{C} 6$. Then, the presence of eight chambers assures plastic particle separation even if a chamber should be filled with the settled material allowing the following chambers to become effective in the separation process. 


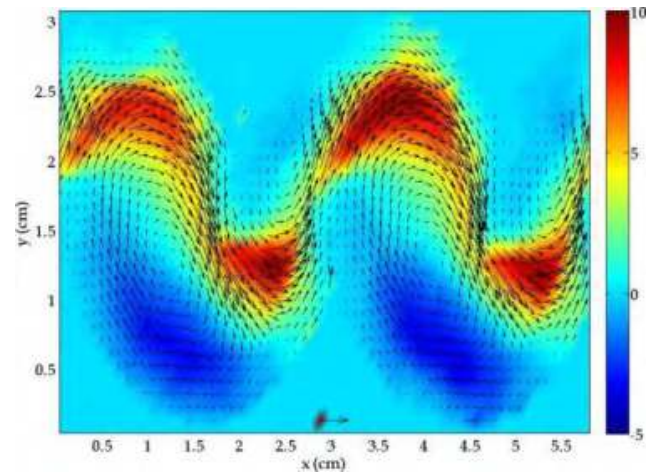

(a)

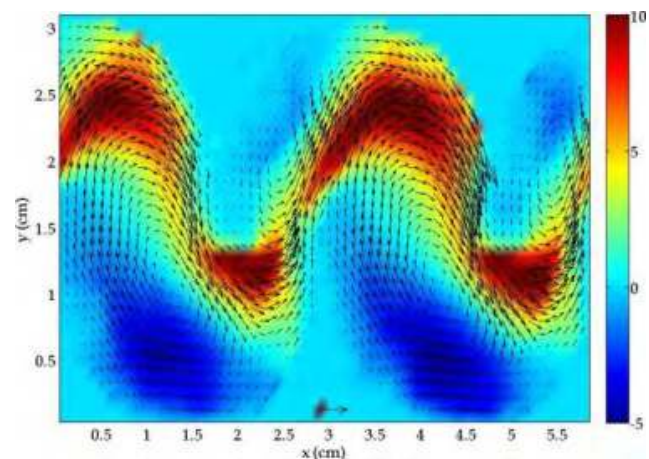

(c)

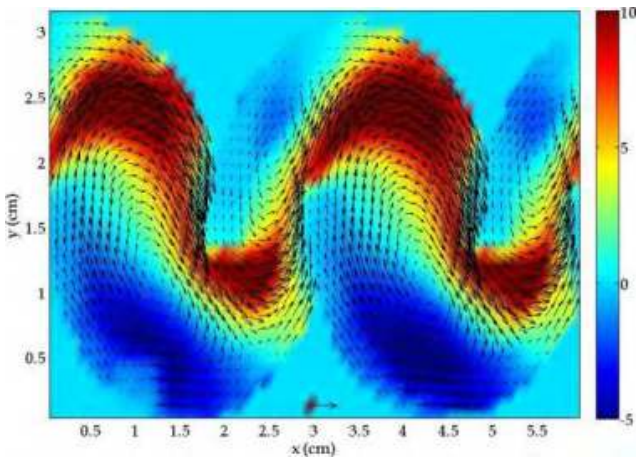

(e)

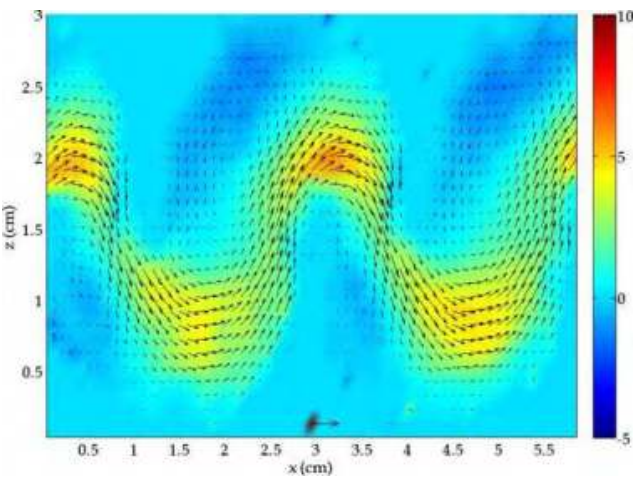

(b)

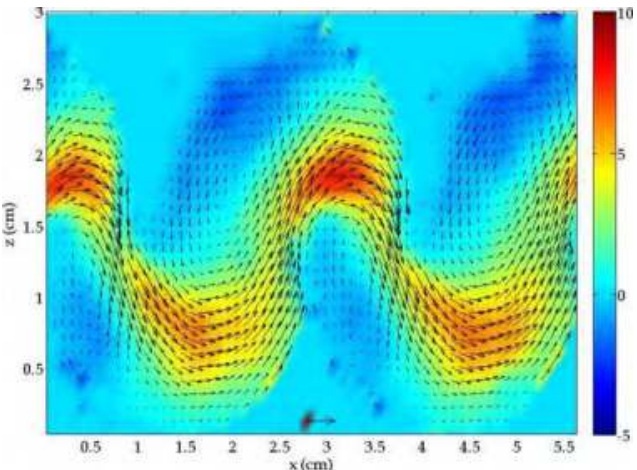

(d)

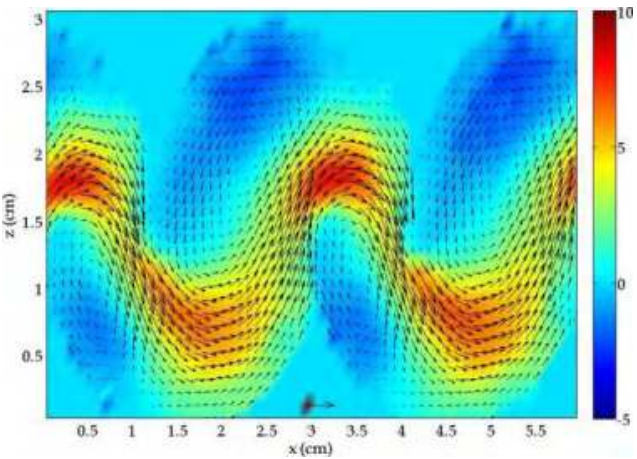

(f)

Fig. 3. Eulerian velocity field within $\mathrm{C} 3$ and $\mathrm{C} 4$ overlapped to the horizontal velocity component represented as colormap for Arrangement A at (a) Q1, (b) Q3, (c) Q5 and Arrangement B at (d) Q1, (e) Q3,(f) Q5. The reference velocity vector is equal to $10 \mathrm{~cm} / \mathrm{s}$ 


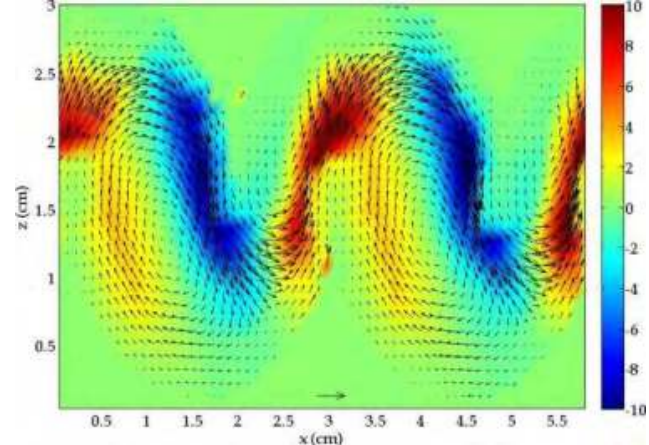

(a)

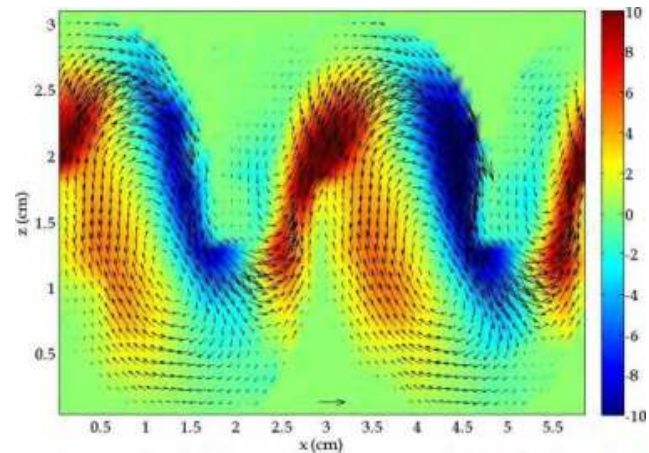

(c)

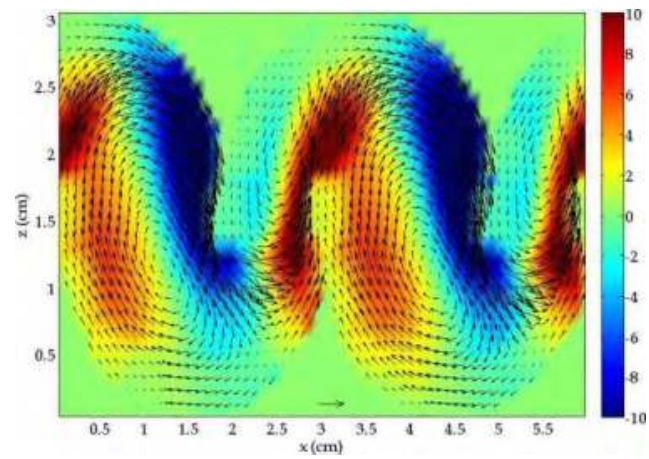

(e)

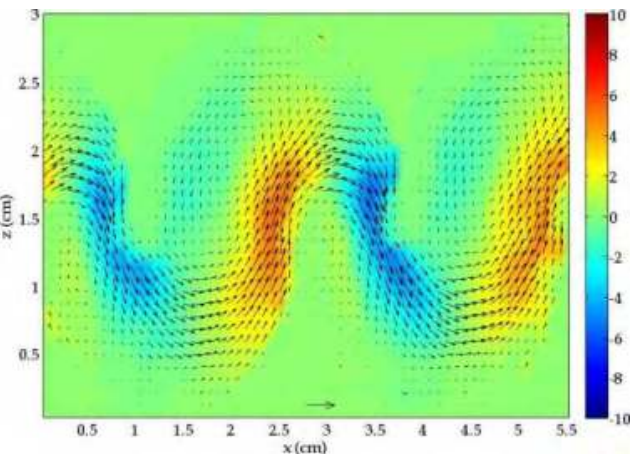

(b)

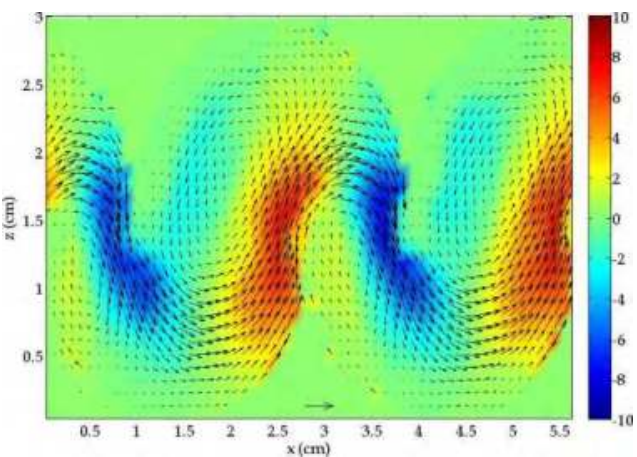

(d)

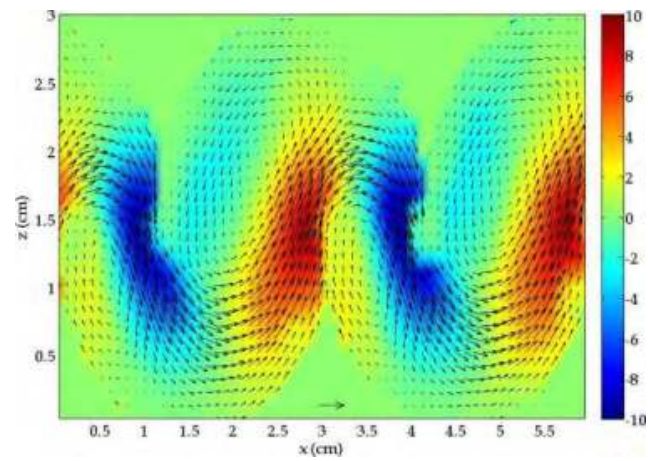

(f)

Fig. 4. Eulerian velocity field within C3 and C4 overlapped to the vertical velocity component represented as colormap for Arrangement A at (a) Q1, (b) Q3, (c) Q5 and Arrangement B at (d) Q1, (e) Q3,(f) Q5. The reference velocity vector is equal to $10 \mathrm{~cm} / \mathrm{s}$ 
Arrangement A

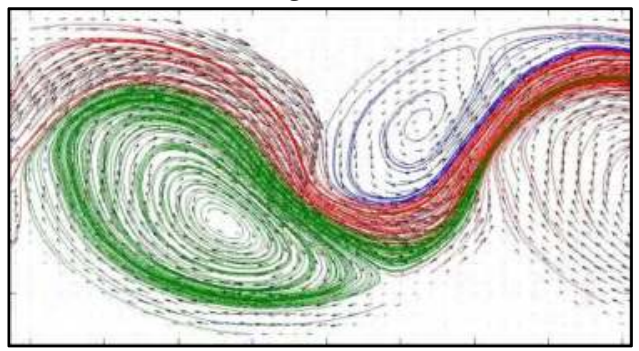

Arrangement B

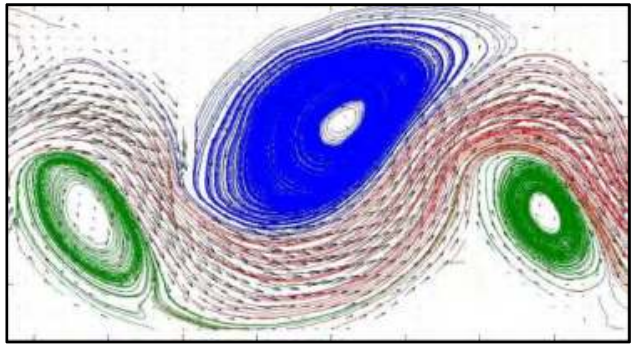

Fig. 5. Velocity field overlapped to the streamlines for Arrangement (a) A and (b) B. The difference in fluid elevation between the tank and the center of the Multidune inlet nozzles is set to Q3

Figure 6 presents the vorticity field computed for both Arrangements and for Q1, Q3 and Q5. The difficulty in identifying vortical structured by examining just $\nabla \times \mathbf{u}$ should be clear from those figures. On the other hand, Figure 7 presents the complex part of the first eigenvalue of the velocity gradient tensor for both Arrangements and the difference in fluid elevation between the tank and the center of the Multidune inlet nozzles is set to Q5. It should be noted as the areas where the first (and the second as well, not shown) presents a complex value is within recirculation areas. It is then confirmed as the criteria previously mentioned for identifying vortical structures can be used for this experimental investigation.

\section{Separation efficacy}

Experimental tests were executed utilising four samples of plastics, in order to investigate efficiency and capability in differentiating trajectories according to plastic typologies and fluid-dynamic properties of the Multidune with both Arrangement A and B (Figure 1). The samples were composed with particles of different plastic material and size, as described in Table 2.

The tank heights and the corresponding flowrates utilized in the experimental tests were Q1, Q3 and Q5, as reported in the previous Table 1.

\begin{tabular}{|c|c|c|c|c|}
\hline & $\begin{array}{c}\text { Sample } \\
\text { material }\end{array}$ & Mean density & $\begin{array}{c}\text { Particle size } \\
\text { distribution }\end{array}$ & Weight \\
\hline Sample & & $\left(\mathrm{g} / \mathrm{cm}^{3}\right)$ & $(\mathrm{mm})$ & $(\mathrm{g})$ \\
\hline MONO.2 & Brown plastic & 1.416 & $0.85-1.00$ & 1.5 \\
\hline MONO.3 & Green plastic & 1.353 & $0.85-1.00$ & 1.5 \\
\hline MONO.4 & Green plastic & 1.353 & $1.70-2.00$ & 2.5 \\
\hline MONO.5 & Red plastic & 1.143 & $1.70-2.00$ & 2.5 \\
\hline
\end{tabular}

Table 2. Composition of samples utilized in separation tests with Arrangements A and B. 


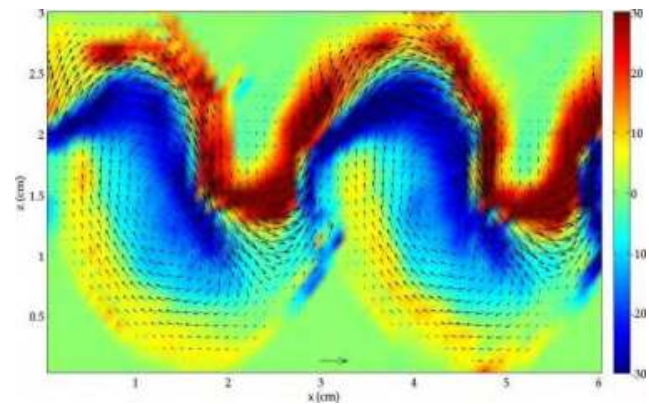

(a)

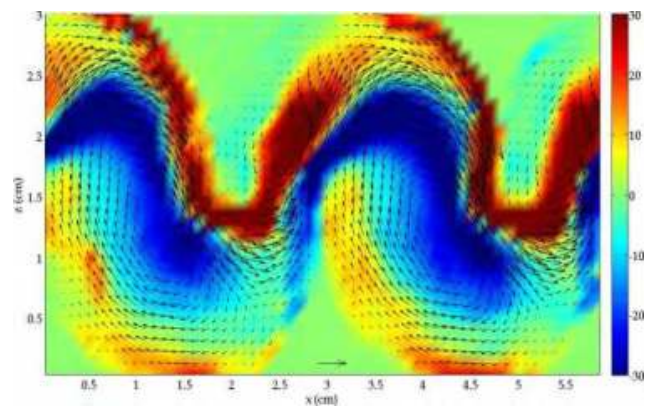

(c)

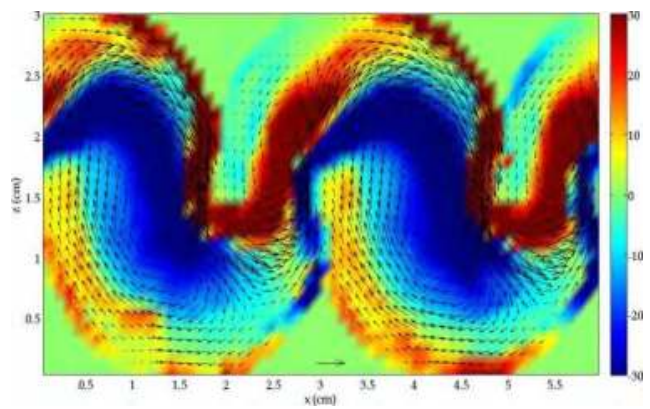

(e)

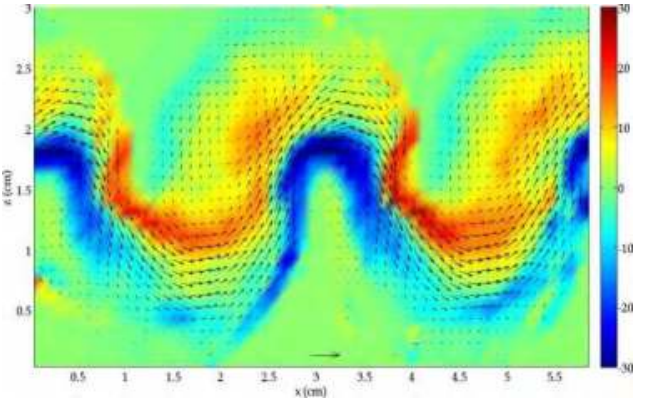

(b)

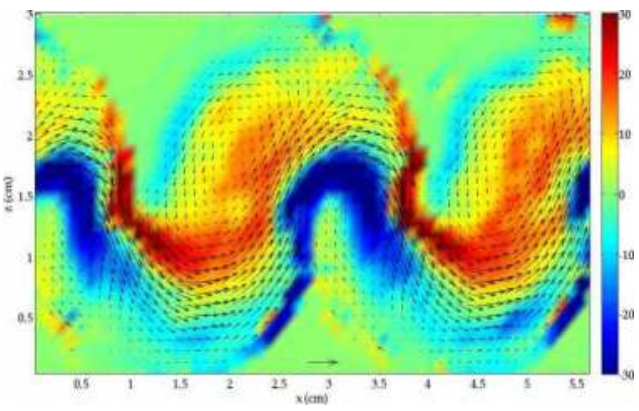

(d)

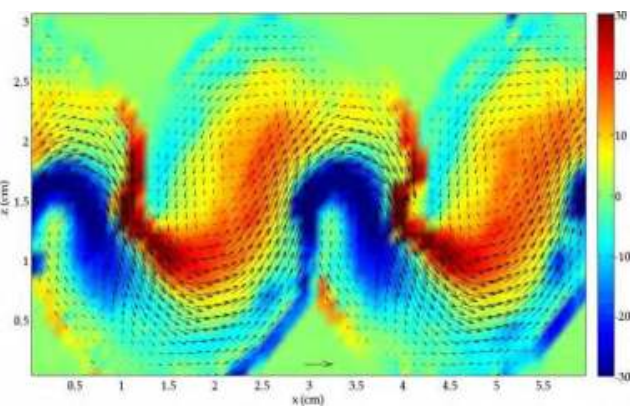

(f)

Fig. 6. Eulerian velocity field within $\mathrm{C} 3$ and $\mathrm{C} 4$ overlapped to the vorticity field represented as colormap for Arrangement A at (a) Q1, (b) Q3, (c) Q5 and Arrangement B at (d) Q1, (e) Q3, (f) Q5. The reference velocity vector is equal to $10 \mathrm{~cm} / \mathrm{s}$

The results of the tests on plastic samples suggested that the system may be able to separate different types of plastics in a mixture imposing an appropriate hydraulic head. In the following further remarks are given about the results referring to the adopted Arrangement. 


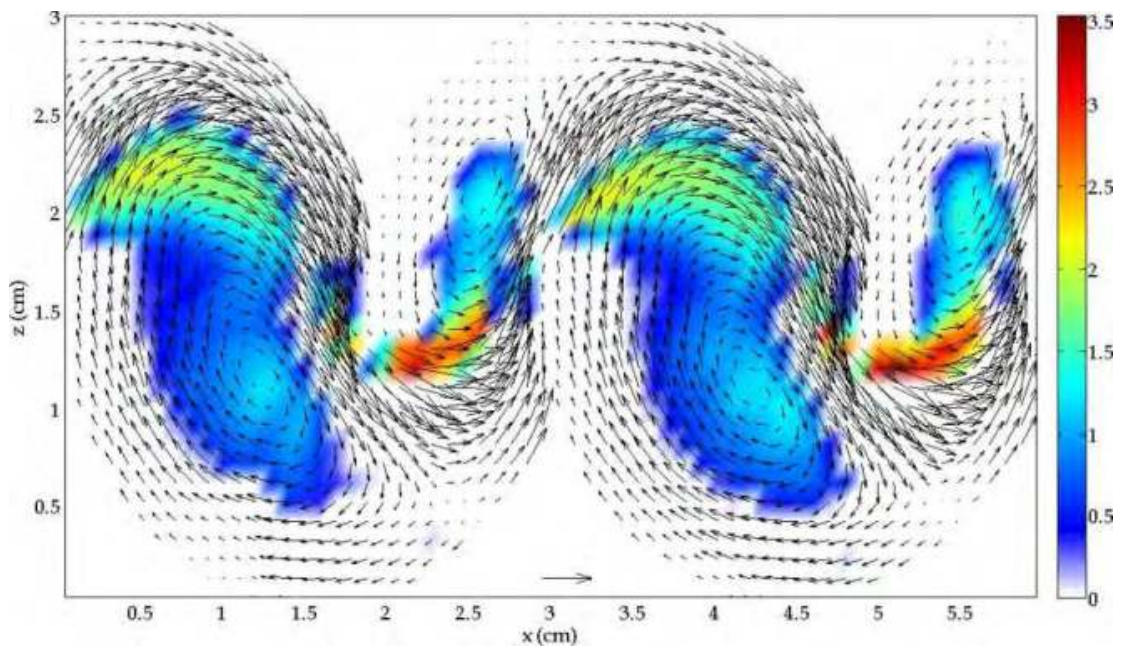

(a)

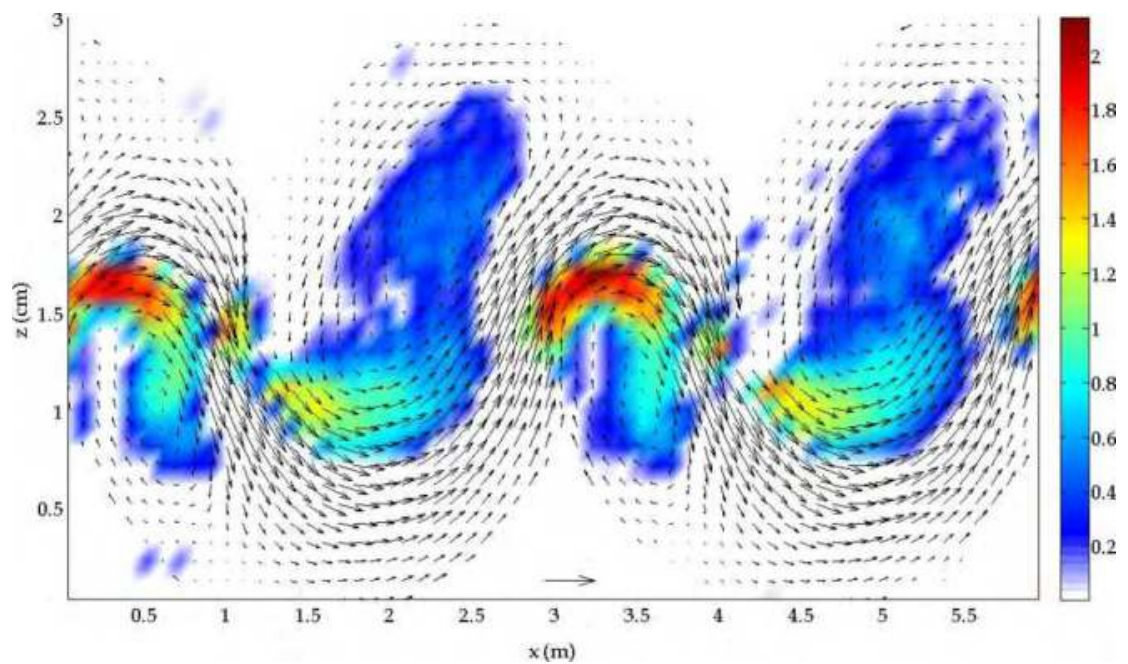

(b)

Fig. 7. Velocity field overlapped to the complex part of the first eigenvalue of the velocity gradient tensor for Arrangement (a) A and (b) B. The difference in fluid elevation between the tank and the center of the Multidune inlet nozzles is set to Q5

\subsection{Results with arrangement $A$}

Figure 8 shows the results of the experimental tests on plastic samples at each adopted flowrate, reporting the percentage in weight of the materials recovered from each chamber (C1-C8) and expelled through the output nozzles (Exp). 


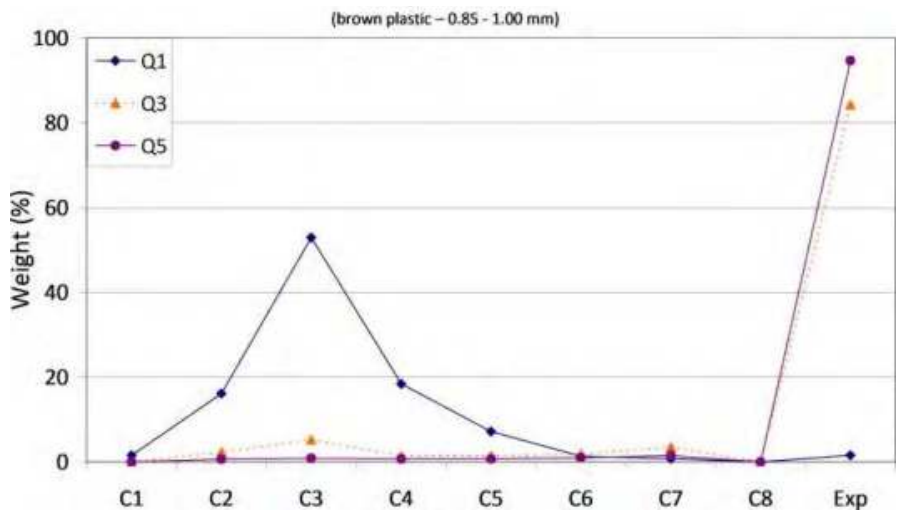

Sample MONO.2

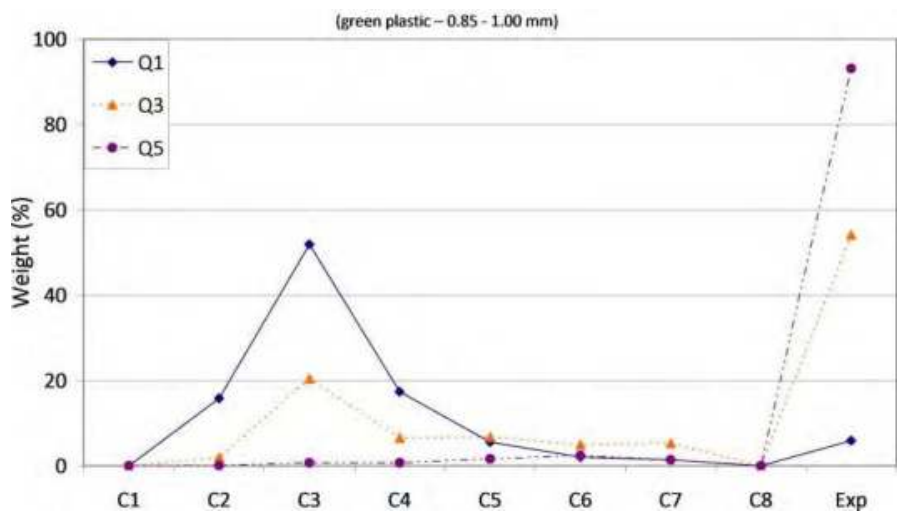

Sample MONO.3

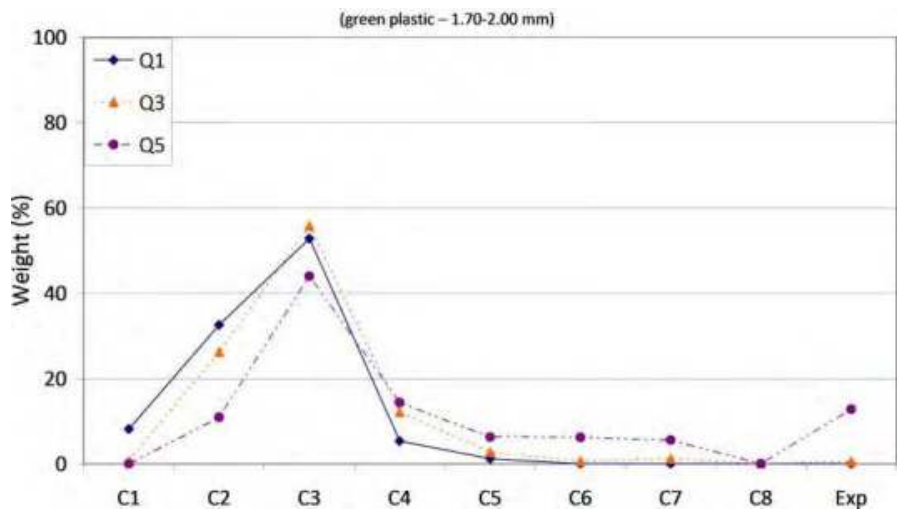

Sample MONO.4 


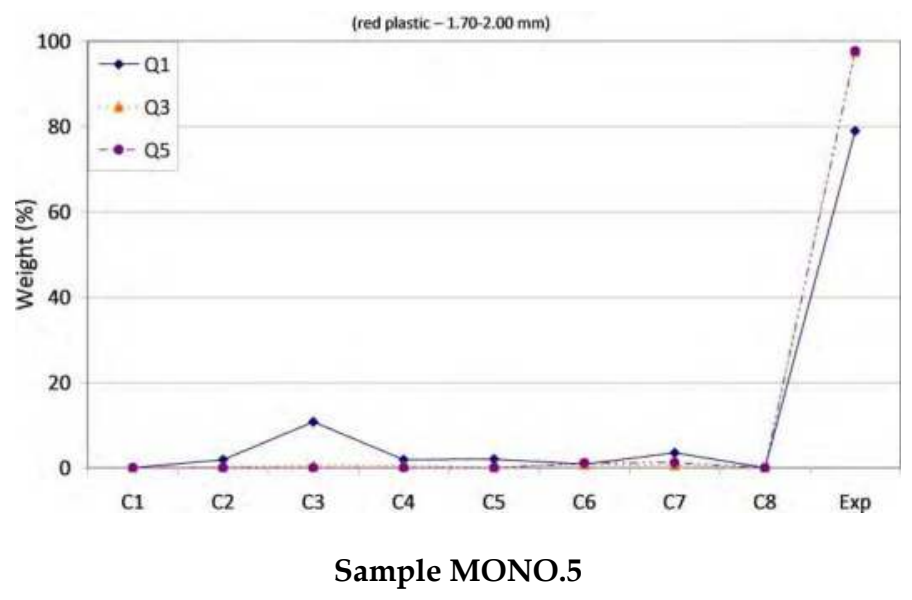

Fig. 8. Results of the experimental tests on mono-material samples at each adopted hydraulic head with Arrangement A

It is evident as the highest hydraulic heads (Q3 and Q5) involve high flowrates, and, therefore, high velocities that sweep away all the small-size particles $(0.85-1.00 \mathrm{~mm}$, samples MONO.2 and MONO.3), without achieving satisfactory differences in respective trajectories.

On the other hand, with the lowest hydraulic head (Q1), the heaviest particles (samples MONO.2, MONO.3 and MONO.4) tend to settle into the apparatus, while the lightest ones (sample MONO.5) tend to be expelled through the output nozzle, regardless of particle size. In this case, a separation of the different types of plastics is possible, thanks to the differentiation of trajectories.

The more selective chamber is the third one, in which the greatest amount of settled material is accumulated, independently from applied flowrate. In the chambers C1 and C8, only few particles settled due to the presence of input and output nozzles, respectively.

Green and brown plastic particles sized 0.85-1.00 mm (samples MONO.2 and MONO.3) show the same behaviour, so a separation seems not possible for each tested operative condition.

Also in the case of coarser particles (samples MONO.4 and MONO.5), the particle settling in the chambers shows the same trend, being C 3 the chamber in which mainly the plastic particles settle regardless of the applied hydraulic head, while $\mathrm{C} 1$ and $\mathrm{C} 8$ remain almost empty.

The green plastic particles sized 1.70-2.00 mm (sample MONO.4) tend to settle in the apparatus, with slight differences in the distribution in the chambers by increasing the hydraulic head; in particular, the particles shift from $\mathrm{C} 2$ to $\mathrm{C} 3$, and then from $\mathrm{C} 3$ to the following chambers.

The red plastic particles sized 1.70-2.00 mm (sample MONO.5) tend to be expelled from the Multidune. Only at the lowest hydraulic head, a significant amount of material settles in the C3.

Therefore, a different behaviour of the samples sized 1.70-2.00 $\mathrm{mm}$ for each tested hydraulic head was observed, so a separation between them seems possible to be achieved. 


\subsection{Results with arrangement B}

Analogous experimental tests have been executed with the Arrangement B of the Multidune (Figure 1). As previously commented, the only difference in the operative procedure was the choice of the output nozzles to be opened. Firstly, the same three output nozzles as in Arrangement A were opened (O2, $\mathrm{O} 4$ and $\mathrm{O} 6)$, but all the samples were expelled out because the high flowrate determined too high flow velocity, in each operative condition. Considering such results, the experiments were carried out by opening only one output nozzle (O3), to lower flowrate and velocity.

Figure 9 shows the results of the experimental tests at each adopted flowrate, in terms of percentage in weight of the materials recovered from each chamber (C1-C8) and expelled through the output nozzle (Exp).

In Arrangement B, it is confirmed as the small-size particles $(0.85-1.00 \mathrm{~mm}$, samples MONO.2 and MONO.3) are quite totally expelled from Multidune imposing the highest hydraulic heads (Q3 and Q5): so, in these operative conditions the trajectories of the two types of plastics are similar.

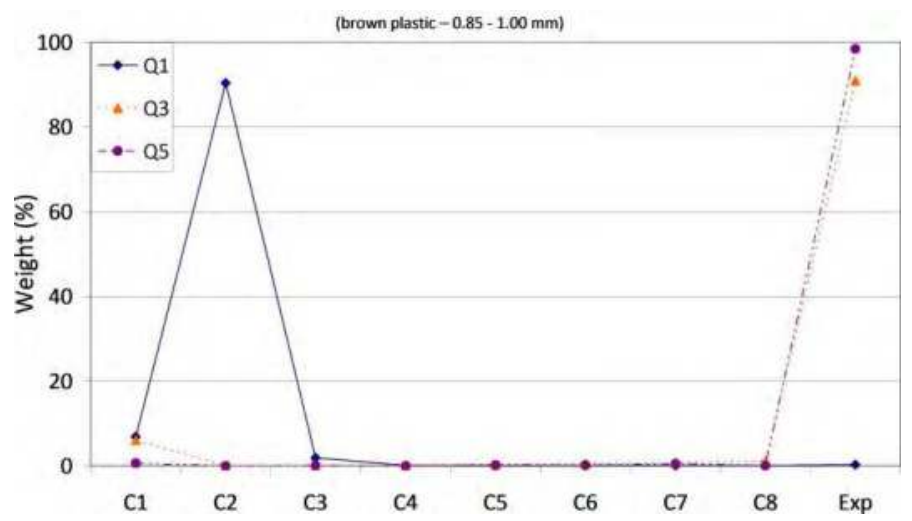

Sample MONO.2

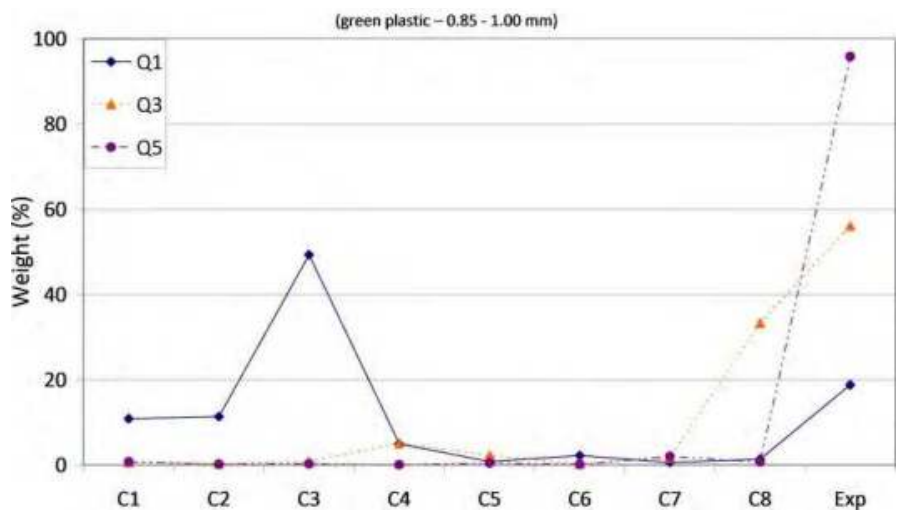

Sample MONO.3 


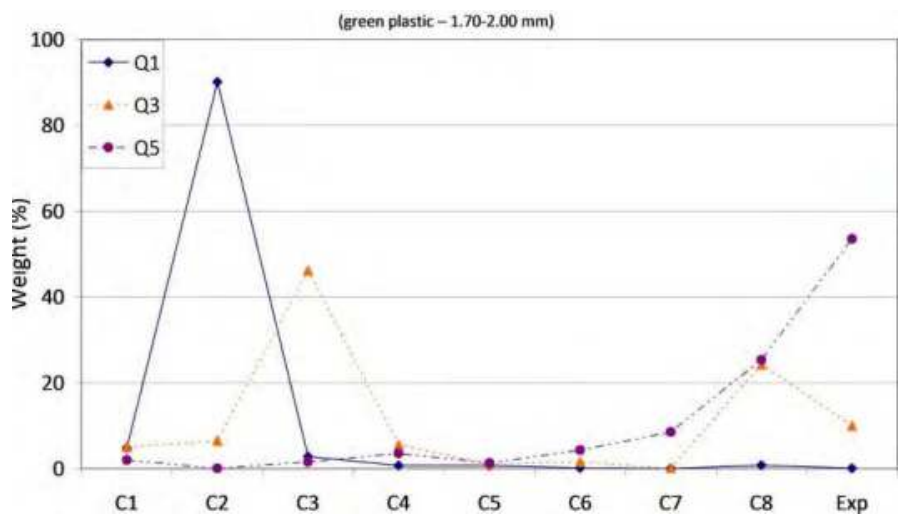

Sample MONO.4

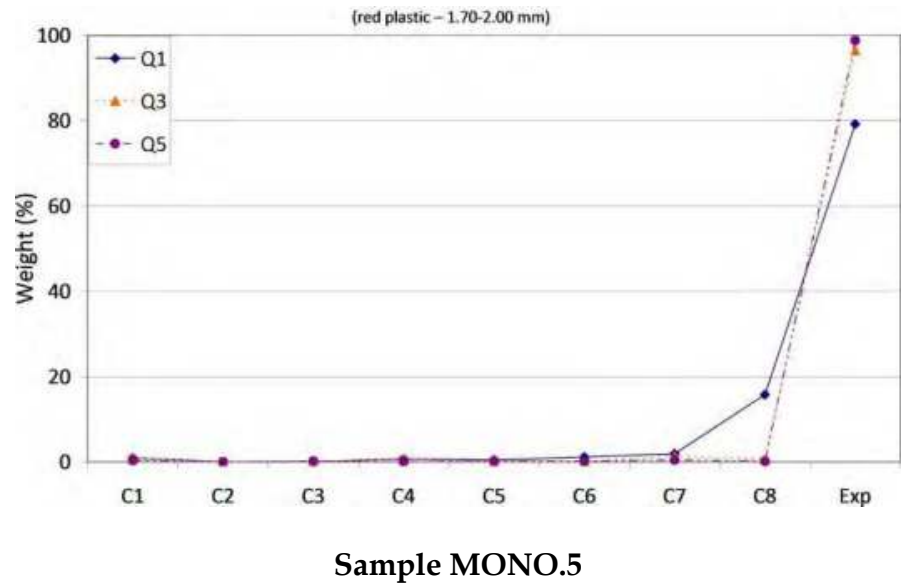

Fig. 9. Results of the experimental tests on mono-material samples at each adopted hydraulic head with Arrangement B

A separation seems to be possible at the lowest hydraulic head (Q1): actually, the heaviest particles (samples MONO.2, MONO.3 and MONO.4) tend to settle into the apparatus, while the lightest ones (sample MONO.5) tend to be expelled through the output nozzle, regardless of particle size.

The distribution of settled particles into the apparatus is more complex in Arrangement B than in Arrangement A. Particles path is in relation with the hydraulic head: as the hydraulic head increases, the particles tend to settled in the chambers closer to the output nozzles, consequently there is not a specific chamber (as C3 in Arrangement A) where particles are mainly accumulated.

The results of tests with Arrangement B confirm that it is possible to separate brown and green particles sized 0.85-1.00 mm (samples MONO.2 and MONO.3) imposing low flowrates, while green and red plastic particles of larger size (samples MONO.4 and MONO.5) can be separated at higher flowrates. 


\section{Conclusion}

The high temporal and spatial resolution technologies employed for the reconstruction of the fluid-dynamic field inside the Multidune allows recognizing the flow field within the apparatus with both arrangements is characterized by three areas: the main transport current and, in each chamber, recirculation areas above and below. The fluid-dynamic behaviour is substantially similar in each chamber but in the first and the last one (C1 and C8), where the inlet and outlet nozzles prevent the formation of similar vortical structures. With Arrangement A, the characteristic velocity of the principal current appears significantly larger than the velocity within the upper and lower recirculation areas; this aspect is amplified with increasing hydraulic head at the apparatus inlet. With Arrangement $\mathrm{B}$ they appear more comparable. With both arrangements, the increase of the hydraulic head augments the transport effectiveness of the main current without improving the capture feasibility of both the upper and lower recirculation zones. The apparatus will then lose its effectiveness in separating plastic particles increasing both the hydraulic head and the transiting flowrate. Furthermore, with both arrangements, C3, C4, C5 and C6 present analogous recirculation areas. The same circumstance occurs at each flowrate establishing in the apparatus. Then, the presence of eight chambers assures plastic particle separation even if a chamber should be filled with the settled material allowing the following chambers to become effective in the separation process.

\section{Acknowledgments}

The authors would like to acknowledge Dr. Leonardo Cherubini and Dr. Emanuela Lupo for their contribution during the experiments.

\section{References}

De Sena G., Nardi C., Cenedese A., La Marca F., Massacci P., Moroni M. (2008). The Hydraulic Separator Multidune: Preliminary Tests on Fluid-Dynamic Features and Plastic Separation Feasibility. Waste Management 29(9), 1560-1571.

Hussain F. (1986). Coherent structures and turbulence, J. Fluid Mech. 173, 303.

Joeng G., Hussain F. (1995). On the identification of a vortex, J. Fluid Mech. 285, 69.

La Marca F., Moroni M., Cherubini L., Lupo E., Cenedese A. (2011). Recycling of plastic waste via the hydraulic separator Multidune. Waste Management, ISSN: 0956-053X, (submitted).

Moroni M., Cenedese A. (2005). Comparison among feature tracking and more consolidated velocimetry image analysis techniques in a fully developed turbulent channel flow. Measurement Science and Technology 16, 2307-2322.

Moroni M., Kleinfelter N., Cushman J.H. (2008). Alternative Measures of Dispersion Applied to Flow in a Convoluted Channel. Advances in Water Resources 32(5), 737-749.

Moroni M., La Marca F., Cherubini L., Cenedese A. (2011). Recovering plastics via the hydraulic separator Multidune: flow analysis and efficiency tests. International Journal of Environmental Science and Technology, ISSN: 1735-1472, (submitted).

PlasticsEurope (2007a). An analysis of plastics production, demand and recovery for 2005 in Europe. PlasticsEurope, $21 \mathrm{pp}$.

PlasticsEurope (2007b). Press Release 9 May 2007, 1 p. Association of Plastics Manufacturers in Europe (AISBL), Brussels, Belgium. 


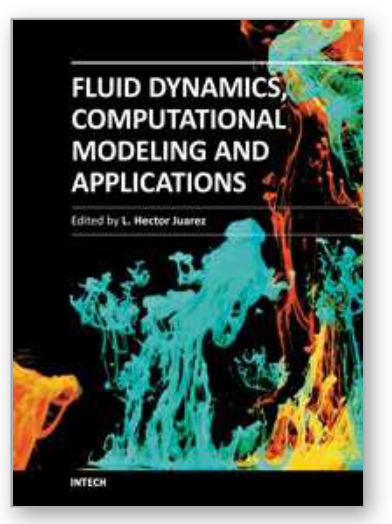

\author{
Fluid Dynamics, Computational Modeling and Applications \\ Edited by Dr. L. Hector Juarez
}

ISBN 978-953-51-0052-2

Hard cover, 660 pages

Publisher InTech

Published online 24, February, 2012

Published in print edition February, 2012

The content of this book covers several up-to-date topics in fluid dynamics, computational modeling and its applications, and it is intended to serve as a general reference for scientists, engineers, and graduate students. The book is comprised of 30 chapters divided into 5 parts, which include: winds, building and risk prevention; multiphase flow, structures and gases; heat transfer, combustion and energy; medical and biomechanical applications; and other important themes. This book also provides a comprehensive overview of computational fluid dynamics and applications, without excluding experimental and theoretical aspects.

\title{
How to reference
}

In order to correctly reference this scholarly work, feel free to copy and paste the following:

Floriana La Marca, Monica Moroni and Antonio Cenedese (2012). Fluid-Dynamic Characterization and Efficiency Analysis in Plastic Separation of the Hydraulic Separator Multidune, Fluid Dynamics, Computational Modeling and Applications, Dr. L. Hector Juarez (Ed.), ISBN: 978-953-51-0052-2, InTech, Available from: http://www.intechopen.com/books/fluid-dynamics-computational-modeling-and-applications/fluid-dynamiccharacterization-and-efficiency-analysis-in-plastic-separation-of-the-hydraulic-separa

\section{INTECH}

open science | open minds

\section{InTech Europe}

University Campus STeP Ri

Slavka Krautzeka 83/A

51000 Rijeka, Croatia

Phone: +385 (51) 770447

Fax: +385 (51) 686166

www.intechopen.com

\section{InTech China}

Unit 405, Office Block, Hotel Equatorial Shanghai

No.65, Yan An Road (West), Shanghai, 200040, China 中国上海市延安西路65号上海国际贵都大饭店办公楼 405 单元

Phone: +86-21-62489820

Fax: $+86-21-62489821$ 
(C) 2012 The Author(s). Licensee IntechOpen. This is an open access article distributed under the terms of the Creative Commons Attribution 3.0 License, which permits unrestricted use, distribution, and reproduction in any medium, provided the original work is properly cited. 